\title{
Potential chemoprevention of diethylnitrosamine-initiated and 2-acetylaminofluorene- promoted hepatocarcinogenesis by zerumbone from the rhizomes of the subtropical ginger (Zingiber zerumbet)
}

\begin{abstract}
Zerumbone (ZER), a monosesquiterpene found in the subtropical ginger (Zingiber zerumbet Smith), possesses antiproliferative properties to several cancer cells lines, including the cervical, skin and colon cancers. In this study, the antitumourigenic effects of ZER were assessed in rats induced to develop liver cancer with a single intraperitoneal injection of diethylnitrosamine (DEN, $200 \mathrm{mg} / \mathrm{kg}$ ) and dietary 2-acetylaminofluorene (AAF) $(0.02 \%)$. The rats also received intraperitoneal ZER injections at 15,30 or $60 \mathrm{mg} / \mathrm{kg}$ body wt. twice a week for 11 weeks, beginning week four post-DEN injection. The hepatocytes of positive control (DEN/AAF) rats were smaller with larger hyperchromatic nuclei than normal, showing cytoplasmic granulation and intracytoplasmic violaceous material, which were characteristics of hepatocarcinogenesis. Histopathological evaluations showed that ZER protects the rat liver from the carcinogenic effects of DEN and AAF. Serum alanine transaminase (ALT), aspartate transaminase (AST), alkaline phosphatase (AP) and alphafetoprotein (AFP) were significantly lower $(\mathrm{P}<0.05)$ in ZER-treated than untreated rats with liver cancer. The liver malondialdehyde (MDA) concentrations significantly $(\mathrm{P}<0.05)$ increased in the untreated DEN/AAF rats indicating hepatic lipid peroxidation. There was also significant $(\mathrm{P}<0.05)$ reduction in the hepatic tissue glutathione $(\mathrm{GSH})$ concentrations. The liver sections of untreated DEN/AAF rats also showed abundant proliferating cell nuclear antigen (PCNA), while in ZER-treated rats the expression of this antigen was significantly $(\mathrm{P}<0.05)$ lowered. By the TUNEL assay, there were significantly $(\mathrm{P}<0.05)$ higher numbers of apoptotic cells in DEN/AAF rats treated with ZER than those untreated. Zerumbone treatment had also increased Bax and decreased Bcl-2 protein expression in the livers of DEN/AAF rats, which suggested increased apoptosis. Even after 11 weeks of ZER treatment, there was no evidence of abnormality in the liver of normal rats. This study suggests that ZER reduces oxidative stress, inhibits proliferation, induces mitochondriaregulated apoptosis, thus minimising DEN/AAF-induced carcinogenesis in rat liver. Therefore, ZER has great potential in the treatment of liver cancers.
\end{abstract}

Keyword: Zerumbone, Chemical carcinogenesis, Hepatocellular carcinoma, Antiproliferative, Apoptosis, Oxidative stress 